\title{
Quality of ultrasonography reporting and factors associated with selection of imaging modality for uterine fibroids in Canada: results from a prospective cohort registry
}

\author{
Olga Bougie MD MPH, Mohamed A. Bedaiwy MD PhD, Philippe Laberge MD, Gerald Lebovic PhD, \\ Nicholas Leyland MD MHCM, Mostafa Atri MD, Ally Murji MD MPH; for the CAPTURE Steering \\ Committee
}

\section{Abstract}

Background: Uterine fibroids are common in women and their management is heavily influenced by information gathered through imaging. We aimed to evaluate the type and quality of imaging performed for assessment of uterine fibroids in Canada.

Methods: Starting in July 2015, premenopausal women with symptomatic fibroids were enrolled in a prospective, noninterventional, observational registry (Canadian Women With Uterine Fibroids Registry [CAPTURE]) that included 19 Canadian sites. Clinical characteristics were extracted from the baseline visit. We evaluated the association between demographic and clinical variables of interest with regard to imaging type using unadjusted and adjusted logistic regression models.

Results: Of 1493 women, 1148 had ultrasonography, 135 had magnetic resonance imaging (MRI), 80 had other types of imaging and 130 did not have imaging reported within 12 months of the baseline visit. After adjusting for demographic and clinical characteristics, patients who underwent MRI had larger fibroids (odds ratio [OR] per 1-cm increase 1.11, 95\% confidence interval [CI] 1.05-1.17) and more numerous fibroids ( 1 v. > 1; OR $1.74,95 \% \mathrm{Cl} 1.14-2.64$ ) compared with those who underwent ultrasonography only. For ultrasonography reporting, quality criteria were met for 268 of 1148 patients (23.3\%). There was a difference in the quality of reporting among the 19 sites $(p<0.001)$. Logistic regression model accounting for within-site variability showed that reporting results from ultrasonography in the province of Quebec were less likely to meet all quality criteria (OR $0.20,95 \% \mathrm{Cl} 0.06-0.66)$ and those from sites in more populated cities ( $\geq 400000$ inhabitants) were more likely to do so (OR 6.15, 95\% Cl 2.20-17.18).

Interpretation: We determined that imaging modality for fibroids is associated with patient characteristics. The quality of reporting results for ultrasonography of fibroids in Canada falls short of internationally endorsed guidelines and needs improvement. Study registration: ClinicalTrials.gov, no. NCT02580578

[ terine fibroids are benign smooth muscle tumours with a prevalence of up to $70 \%-80 \%$ in women by the age of 50 years. ${ }^{1}$ About half of women with uterine fibroids will experience symptoms of abnormal uterine bleeding, pressure and reproductive issues. ${ }^{2}$ Fibroids can vary in size, shape, location and number among patients. This topographical information is needed to help guide treatment decisions and is particularly important for surgical planning. ${ }^{3}$ Although ultrasonography is the mainstay for the diagnosis and monitoring of uterine fibroids, magnetic resonance imaging (MRI) can also be used. ${ }^{4}$

There are no clinical practice guidelines to help determine when an MRI should be ordered to characterize fibroids, and guidelines on the management of fibroids de-emphasize the role of MRI. ${ }^{5}$ The Society of Obstetricians and Gynaecologists of Canada clinical practice guideline on the management of uterine fibroids advises that while ultrasonography imaging is the most widely used imaging modality because of its availability, ease of use and cost-effectiveness, MRI is more accurate in characterizing fibroids in terms of size, location, number and perfusion. ${ }^{3}$

Competing interests: Olga Bougie has presented at continuing medical education events sponsored by Allergan Canada, Bayer, Hologic and AbbVie. Mohamed Bedaiwy is a member of the advisory boards for AbbVie and Allergan. Philippe Laberge is the Chair of the CAPTURE study and a consultant for Allergan, AbbVie, Hologic, Medtronic, Minerva and Olympus. Nicholas Leyland has received research support from Allergan and AbbVie. Ally Murji is a member of Speaker Bureaus and advisory boards for AbbVie, Allergan, Bayer and Hologic. No other competing interests were declared.

This article has been peer reviewed.

Correspondence to: Olga Bougie, olga.bougie@kingstonhsc.ca CMAJ Open 2020. DOI:10.9778/cmajo.20200004 
The decision to order MRI is often based on characteristics of patients and providers, and likely to be dependent on the practice setting. Little is known about the real-world choices for fibroid-imaging modalities. Regardless of the choice of modality, it is essential that imaging provides the clinician with details on fibroid characteristics to help guide the management approach. Hence, the quality of imaging may be even more important than the modality itself.

In 2015, the International Society of Ultrasound in Obstetrics and Gynecology endorsed the Morphological Uterus Sonographic Assessment (MUSA) consensus statement, which described the sonographic features and terminology for reporting on uterine fibroids. ${ }^{6}$ This document called for standardized reporting to reduce the variability in the evaluation of fibroids. The goal of systematic standardization was to improve the quality of reporting, and thereby optimize clinical management of this condition. The uptake of this guideline in clinical settings is unknown.

A prospective, noninterventional, multisite, observational registry of premenopausal women with symptomatic uterine fibroids (Canadian Women with Uterine Fibroids Registry [CAPTURE]) was established in Canada in 2015. This registry provides an opportunity to describe practice patterns in the diagnosis and management of fibroids across diverse geographic and practice settings. The study had 2 objectives: to evaluate the quality of and variation in ultrasonography reporting within the Canadian health care system and to describe factors associated with the use of MRI to evaluate uterine fibroids.

\section{Methods}

\section{Setting}

The CAPTURE registry comprised a cohort of women with symptomatic uterine fibroids from 19 study sites across Canada (ClinicalTrials.gov no. NCT02580578). The methods used to develop the registry were published previously. ${ }^{7}$ The study sites were a mix of academic and community centres.

\section{Study design}

We conducted a prospective, noninterventional cohort study in which physicians were not required to perform any medical procedure that was outside their routine clinical practice. Overall coordination of the registry was led by a steering committee that included 6 academic and 1 community gynecologists. This committee established the registry database and protocol and appointed a scientific committee for data analysis and interpretation. All investigations were ordered at the physicians' discretion and performed and interpreted at various clinical practice locations based on provider and patient preference.

Inclusion criteria were clinically premenopausal female patients aged 18 years or older with symptoms associated with uterine fibroids who were being observed (watchful waiting), currently being treated or starting treatment (drug intervention, procedure intervention or a combination of both). Patients were required to provide written, informed consent before or at the initial study visit. Exclusion criteria included known or suspected substantial pelvic pathology not associated with uterine fibroids and patients undergoing an emergency hysterectomy at the initial visit. A description of baseline cohort characteristics was previously published and showed that patients included in the cohort represented women with varied demographic and socioeconomic characteristics, in keeping with the diversity of the Canadian population. ${ }^{8}$

\section{Sources of data}

We recorded data pertaining to imaging at the initial study visit. Imaging was ordered as part of routine clinical practice and was not affected by patient participation in the study. We extracted imaging type and uterine and fibroid characteristics described in the imaging reports from the patient chart. Imaging for uterine fibroids performed within 12 months of the baseline visit (either before or after the visit) was recorded in the registry. If this was the only imaging investigation a patient had, the patient was classified as having "ultrasonography only." Otherwise, the patient was classified as "other" for the imaging modality category. A patient could be classified into 1 group only, not multiple groups.

If available, we extracted the following uterine fibroid characteristics from the imaging reports: number of fibroids, diameter of fibroids, fibroid volume, type of fibroid and location of the fibroid. We also extracted patient demographic information, medical history and evaluations of past and current symptomatology for each patient. We used the Uterine Fibroid Symptom and Health-Related Quality of Life questionnaire, ${ }^{9,10}$ and the Aberdeen Menorrhagia Severity Scale (AMSS) bleeding score to obtain baseline measures of patient-reported outcomes. ${ }^{11} \mathrm{We}$ also recorded characteristics of the medical practice in which the patient was seen, including geographic region within Canada (Western Ontario, Central Ontario, Eastern Ontario, Quebec, Western Canada and Eastern Canada), academic versus community practice and city size based on population (we considered a city to be small if it had a population of $<400000$; Appendix 1 , available at www.cmajopen.ca/content/8/3/E506/suppl/DC1).

Data were recorded in the Research Electronic Data CAPTURE database by trained study personnel at each study visit. Data quality assurance included real-time flagging of missing data, flagging of values outside preestablished ranges and quarterly site visits by central research teams to ensure accuracy of data entry for each patient chart.

\section{Quality criteria for ultrasonography reporting}

We assigned each ultrasonography report a quality rating based on 5 criteria that were adapted from the MUSA consensus statement, as described below. ${ }^{6}$

\section{Fibroid number}

If the report mentioned a specific number of fibroids, it met the quality standard. If it reported "multiple" or "unspecified" number of fibroids, then it did not meet the quality standard.

\section{Fibroid dimensions}

If all 3 dimensions of the largest fibroid were reported, the report met the quality standard. 


\section{Uterine dimensions}

If all 3 uterine dimensions or a uterine volume were reported, the report met the quality standard.

\section{Fibroid type}

A report describing any of the following for the largest fibroid met the quality standard: submucosal (International Federation of Gynecology and Obstetrics type 0, 1, 2, unknown type), intramural, subserosal, cervical or pedunculated.

\section{Fibroid location}

A report describing any of the following for the largest fibroid location met the quality standard: anterior, lateral, posterior or fundal.

We considered an ultrasonography report to be of high quality if it met all 5 quality standards.

The MUSA statement refers to imaging of the myometrium in general, not specifically for fibroids. ${ }^{6}$ Some of its recommendations, such as descriptions of the junctional zone, are more pertinent to other uterine diseases, namely adenomyosis. As this was not the focus of our study, we did not extract this information from the ultrasonography reports. As such, we retrieved only the fibroid-specific characteristics using the ultrasonography reporting recommendations from the MUSA statement.

\section{Statistical analysis}

We conducted descriptive analyses of demographic and clinical variables of interest. We summarized continuous data using mean and standard deviation (SD) or median and interquartile range (IQR). Categorical variables were summarized using counts and percentage. Fisher exact tests or $\chi^{2}$ tests, as appropriate, were used to test for unadjusted differences in categorical variables between imaging groups. Parametric or nonparametric $t$ tests, as appropriate, were used to test for unadjusted differences in continuous variables between imaging groups.

We evaluated the association between demographic and clinical variables of interest with regard to imaging type using unadjusted and adjusted logistic regression models. We used a generalized linear mixed model to evaluate associations between hypothesis-generating covariates and the outcome of having quality ultrasonography. This model adjusted for the following characteristics: age; body mass index (BMI); ethnicity; gravidity (any v. none); history of infertility (yes, no or unknown); previous medical or surgery intervention; geographic region; community versus academic centre; and city population size. We placed a random effect in the model to account for correlation arising within clinical site. The median odds ratio (OR), a measure of heterogeneity that is adjusted for patient-level covariates, was computed from the adjusted model. ${ }^{12}$ The sites in Nova Scotia and Newfoundland had few participants; therefore, we could not properly estimate the random effect in the mixed model and the median OR.

\section{Missing data}

There were 1290 eligible participants in the analysis who had an ultrasonography or MRI (eligible participants had only one or the other - we excluded all those who had both). Of those participants, we excluded 7 who had unknown dates of baseline imaging or had imaging more than 1 year before baseline. Therefore, we included 1283 participants in the analysis. In the model, using complete case analysis, we had full data on 1199 of the 1283 participants or about $93.5 \%$ of the participants remained in the analysis. Therefore, we used complete case analysis because few data were missing and we assumed this data to be missing at random.

For the model evaluating the quality of ultrasonography, we included the 1148 participants who had undergone this procedure. We excluded 2 of these participants because we could not determine if they had any previous procedural interventions (i.e., myomectomy or uterine artery embolization). Of the 1146 included in the data set, there were 1128 with all data available, which allowed us to use complete case analysis for this model.

\section{Ethics approval}

Approval was obtained from research ethics boards at each participating study site (Appendix 1).

\section{Results}

Our study included 1493 women from 19 practice sites in Canada. The study sites were distributed as follows: 3 in British Columbia, 2 in Alberta, 1 in Saskatchewan, 7 in Ontario, 4 in Quebec and 2 in Atlantic Canada (Nova Scotia and Newfoundland). For 1148 (76.9\%) of these women, ultrasonography was the only imaging modality recorded. At the baseline visit, 135 (9.0\%) women had an MRI report, 80 (5.4\%) had another imaging modality (i.e., hysterosalpingography or computed topography) and $130(8.7 \%)$ did not have an imaging report. We excluded the latter 2 groups from further analysis. Of the 130 women classified as having no imaging reported at baseline, 104 (80\%) did have an imaging diagnosis of fibroids that was performed more than 12 months before the baseline visit. We excluded these 130 women from further analysis. Baseline characteristics of women who had only ultrasonography or MRI within 12 months of the baseline visit are shown in Table 1.

Compared with patients having only an ultrasonography, participants who had an MRI were more likely to have larger (OR per $1-\mathrm{cm}$ increase in fibroid diameter $1.13,95 \%$ confidence interval [CI $1.07-1.18$ ) and more numerous (OR of $>1$ v. 1 fibroid $1.58,95 \%$ CI $1.05-2.36$ ) fibroids. This trend remained after we adjusted for demographic and clinical characteristics (adjusted OR per 1-cm increase in fibroid diameter $1.09,95 \%$ CI 1.03-1.16, and adjusted OR of > 1 v. 1 fibroid $1.79,95 \%$ CI $1.15-2.78)$. In the unadjusted analysis, older patients were more likely to have an MRI (OR per 5-yr age increase $1.35,95 \%$ CI $0.19-1.53)$. However, after we adjusted for demographic and clinical characteristics, we found that older patients were less likely to have an MRI (OR per 5-yr age increase $0.74,95 \%$ CI 0.64-0.85). Patients who had an MRI reported lower menstrual bleeding scores (OR for a 10 -point increase in AMSS score 0.87, 95\% CI 0.79-0.96, and 
Table 1: Demographic characteristics of participants based on imaging modality

\begin{tabular}{|c|c|c|c|}
\hline \multirow[b]{2}{*}{ Characteristic } & \multicolumn{2}{|c|}{ No. of participants $(\%)^{*}$} & \multirow[b]{2}{*}{$p$ value } \\
\hline & $\begin{array}{l}\text { Ultrasonography } \\
\qquad n=1148\end{array}$ & $\begin{array}{c}\text { MRI } \\
n=135\end{array}$ & \\
\hline Age, yr, mean \pm SD & $43.22 \pm 6.69$ & $40.24 \pm 7.30$ & $<0.001$ \\
\hline Body mass index, mean \pm SD & $27.28 \pm 6.29$ & $27.20 \pm 7.04$ & 0.9 \\
\hline Nulliparous & $483(42.1)$ & $91(67.4)$ & $<0.001$ \\
\hline Nulligravid & $358(31.1)$ & $61(45.1)$ & 0.001 \\
\hline Family history of fibroids, yes v. no or unknown & $401(34.9)$ & $46(34.1)$ & 0.9 \\
\hline Previous procedural intervention for fibroid, yes v. no or unknown & $251(21.8)$ & $29(21.4)$ & 1.0 \\
\hline History of bulk symptoms, yes v. no or unknown & $663(57.7)$ & $90(66.6)$ & 0.06 \\
\hline Maximum fibroid diameter, $\mathrm{mm}$; mean $\pm \mathrm{SD}$ & $75.56 \pm 36.01$ & $90.15 \pm 34.67$ & $<0.001$ \\
\hline \multicolumn{4}{|l|}{ No. of fibroids } \\
\hline 1 & $408(35.5)$ & $35(25.9)$ & \multirow[t]{6}{*}{$<0.001$} \\
\hline 2 & $191(16.6)$ & $20(14.8)$ & \\
\hline 3 & $135(11.7)$ & $6(4.4)$ & \\
\hline 4 & $45(3.9)$ & $5(3.7)$ & \\
\hline$>4$ & $101(8.8)$ & $17(12.6)$ & \\
\hline Multiple or not specified & $268(23.3)$ & $52(38.5)$ & \\
\hline Score for UFS-QOL, mean \pm SD & $50.25 \pm 23.41$ & $46.45 \pm 22.77$ & 0 \\
\hline Score for HRQoL, mean \pm SD & $50.45 \pm 25.23$ & $52.05 \pm 26.39$ & 0.5 \\
\hline Score for AMSS, mean \pm SD & $37.07 \pm 8.89$ & $31.85 \pm 20.74$ & 0.003 \\
\hline Academic centre & $622 \pm 54.2$ & $84 \pm 62.2$ & 0.09 \\
\hline \multicolumn{4}{|l|}{ Region } \\
\hline Western Ontario & $160(13.9)$ & $16(11.8)$ & \multirow[t]{5}{*}{0.076} \\
\hline Eastern Ontario & $131(11.4)$ & $24(17.7)$ & \\
\hline Central Ontario & 264 (22.9) & $29(21.4)$ & \\
\hline Quebec & $267(23.2)$ & $21(15.5)$ & \\
\hline Western Canada & $313(27.2)$ & $42(31.1)$ & \\
\hline Small city size & $437(38.0)$ & $41(30.3)$ & 0.098 \\
\hline
\end{tabular}

adjusted OR 0.89, 95\% CI 0.81-0.99). In the adjusted analysis, we found no difference in the odds of having an MRI based on BMI (OR per 1-unit increase in BMI 1.0, 95\% CI $0.97-1.03$, and adjusted OR $1.02,95 \%$ CI $0.98-1.05$ ), gravidity (>0 v. 0 , OR $0.55,95 \%$ CI $0.38-0.79$, and adjusted OR $0.89,95 \%$ CI $0.59-1.37$ ), infertility (OR $2.08,95 \%$ CI $1.26-$ 3.43 , and adjusted OR $1.11,95 \%$ CI $0.71-1.75)$ or ethnicity/ race $(p=0.02)$.

The quality of ultrasonography reporting is shown in Table 2. Overall, 268 (23.3\%) ultrasonography reports met all 5 quality criteria. Four quality criteria were met by 365 (31.8\%) reports, 3 quality criteria were met by 326 (28.4\%) and 2 quality criteria were met by $162(14.1 \%)$. Twenty-seven $(2.4 \%)$ reports did not meet any quality criteria. The proportion of ultrasonography reports that met each individual quality criterion is depicted in Figure 1.
We used an adjusted generalized linear mixed model that included 1128 participants to evaluate the association of patient and institutional characteristics with receiving ultrasonography that met all 5 quality standards. There were no participant characteristics that were associated with having a high-quality ultrasonography report. However, compared with participants from Central Ontario (referent group), those from Quebec (OR 0.20, 95\% CI 0.06-0.66) were less likely to have a high-quality report. Patients from study sites in more populated cities ( $\geq 400000$ inhabitants) were more likely to receive a high-quality ultrasonography report (OR 6.15, 95\% CI 2.20-17.18).

After we adjusted for institutional and participant characteristics (described above), the median OR across study sites was 1.66. In other words, the median odds of receiving a highquality ultrasonography procedure were 1.66 times greater if 


\begin{tabular}{|lc|}
\hline $\begin{array}{l}\text { Table 2: Quality of reporting of ultrasonography results in } \\
\text { Ontario* }\end{array}$ \\
\hline Quality criterion & $\begin{array}{c}\text { No. of participants (\%) } \\
n=1148\end{array}$ \\
\hline Fibroid number & $880(76.6)$ \\
\hline Meets standard & \\
\hline Fibroid dimensions & $667(58.1)$ \\
\hline Meets standard & $179(15.6)$ \\
\hline 2 dimensions reported & $275(23.9)$ \\
\hline 1 dimension reported & $504(43.9)$ \\
\hline 0 dimensions reported & $3(0.2)$ \\
\hline Uterine dimensions & $5(0.4)$ \\
\hline Meets standard & $636(55.4)$ \\
\hline 2 dimensions reported & $1120(97.5)$ \\
\hline 1 dimension reported & $268(23.3)$ \\
\hline 0 dimensions reported & \\
\hline Fibroid type & \\
\hline Meets standard & \\
\hline Fibroid location & \\
\hline Meets standard & \\
\hline Meeting quality standard for all 5 criteria & \\
\hline${ }^{*}$ Auality criteria are adapted from the Morphological Uterus Sonographic \\
\hline Assessment Consensus statement.
\end{tabular}

the same participant had imaging at 1 random study site as opposed to another. This interhospital variation was not explained by patient characteristics and only partially by region and city size. The logistic regression model above explained $42 \%$ of the observed variation in quality rates and had good discrimination $(c=0.78)$. Similarly, a logistic regression that did not account for variability between sites explained $38 \%$ of the variation and had only slightly lower discrimination $(c=0.75)$.

When we compared the 19 study sites with their rates of high-quality ultrasonography, we found considerable variation. There was a difference $(p<0.0001)$ in the quality of reporting between the 19 sites (best site had 56 of 111 [50.5\%] scans meeting all criteria v. the worst site with 0 of 19 $[0.0 \%])$. The median rate of high-quality ultrasonography reports was 16.8 per 100 ultrasonography procedures (range $0-50.9)$. Figure 2 shows the variation in high-quality ultrasonography across sites.

\section{Interpretation}

We identified substantial limitations in the quality and variability of ultrasonography reporting in Canada. In this prospective cohort of 1493 women who underwent an imaging evaluation for uterine fibroids, we identified that women who had an MRI for evaluation of uterine fibroids had larger and more numerous fibroids. Furthermore, only $23 \%$ of ultrasonography reports met all quality criteria, as recommended by the MUSA guideline. ${ }^{6}$ There was considerable intersite varia- tion in the quality of ultrasonography reports, which was not explained by participant characteristics and only partially by region and city size.

Renumeration for the physician who reported the results from ultrasonography may influence this variability, but we were not able to capture this information through our study. Furthermore, larger volume centres may have access to ultrasonography technicians who can complete the scans and save images for the radiologist to review. Smaller centres may not use technicians, and the physician may be more likely to do the scanning themselves. Since results from ultrasonography may be reported by either gynecologists or radiologists, there may also be variability of reporting based on specialty, although we did not assess this in our study.

While ultrasonography is the first-line imaging modality for uterine fibroids, ${ }^{3}$ our study also aimed to explore whether specific patient or fibroid characteristics were associated with the use of MRI. We found that after adjusting for patient demographics and clinical practice characteristics, MRI was more likely to be obtained in cases of larger and more numerous fibroids. These larger fibroids are more likely to be subserosal or intra-mural in location and less likely to contribute to heavy bleeding and more likely to result in bulk symptoms.

These results are consistent with previously published literature that reported that the capacity of ultrasonography for accurate fibroid mapping falls short of MRI in large $(>375 \mathrm{~mL})$ multi-fibroid ( $>4)$ uteri. ${ }^{4}$ Surgical planning for uterine preserving procedures may be particularly challenging with large and numerous fibroids. Considering that MRI allows better soft tissue contrast, larger field-of-view and multiplanar imaging capabilities, it can be particularly helpful for surgical planning in such cases, as well as assessment of other possible diagnoses and exclusion of malignancy. ${ }^{13}$ Magnetic resonance imaging also allows for the ease of characterization of numerous fibroids at once, which may be quite laborious with ultrasonography.

Owing to the cost differential between these imaging modalities, standardized algorithms that incorporate the costeffectiveness of each modality would be helpful to guide clinicians in their decision to order MRI. We suggest that after a high-quality ultrasonography assessment of fibroids, specific criteria should be used to determine which patients would further benefit from an MRI.

It is sobering that the odds of a Canadian woman with uterine fibroids receiving high-quality ultrasonography were 1.66 times greater if the same patient had imaging at 1 random institution as opposed to another. These findings are reflective of the limited focus on the importance of standardized imaging for the evaluation of uterine fibroids within clinical practice guidelines. ${ }^{3,14}$ Much of the focus of international guidelines on uterine fibroids is on providing guidance on management rather than thorough evaluation of the condition. ${ }^{3,14,15}$ However, accurate diagnosis and assessment of uterine fibroids is essential to guide optimal selection of treatment strategies, particularly since fibroid characteristics are unique between patients. We observed that fibroid number, type and location were more consistently reported accurately than uterine or fibroid size. 


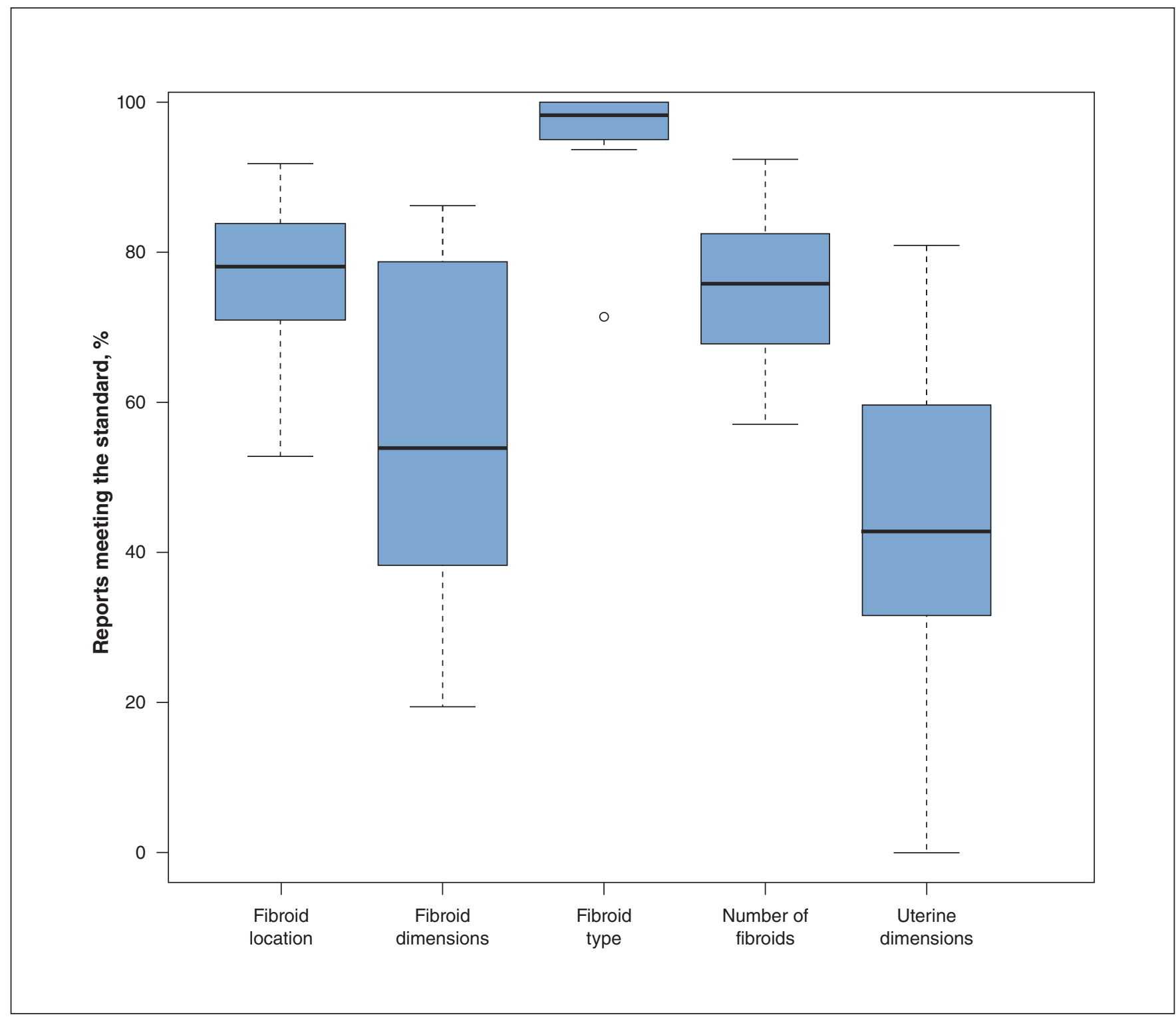

Figure 1: Distribution of ultrasonography reports that met each quality criterion. Note: Bold horizontal bars are the medians, lower and upper horizontal bars of the box are the first and third quartiles, short horizontal lines (whiskers) represent either 1.5 times the interquartile range (IQR) or the minimum and maximum as appropriate. A dot represents an outlier falling outside the whisker $(1.5 \times$ IQR).

It is important to mention that the MUSA recommendations were established by a European team performing high-quality endovaginal ultrasonography, ${ }^{6}$ not transabdominal ultrasonography. In the Canadian context, transvaginal ultrasound may be a second-line examination in many parts of the country. Unfortunately, one of the limitations of the CAPTURE database is that it did not collect data about the route of ultrasonography that was performed. This information is missing because the registry was designed to evaluate long-term clinical and patientreported outcomes in patients with uterine fibroids, rather than the modality or quality of imaging.

This analysis was prompted when we discovered the large variation in ultrasonography reporting in this registry and thought it was important to evaluate the prevalence and extent of this problem with an aim to help improve pelvic imaging for women with fibroids in Canada. The women included in the study were symptomatic from their fibroids, necessitating a gynecology consultation, and over three-quarters had imaging that did not provide clinically important information about their pathology, which is concerning. Irrespective of the current route of sonography, there are no Canadian-specific guidelines or standards for reporting results for ultrasonography of uterine fibroids.

We propose that prompt evaluation of factors influencing imaging quality are necessary. Factors limiting the quality of reporting ultrasonography results may include lack of knowledge, dissemination of imaging practice guidelines, limited training and time or resource restraints, as well as patient characteristics (i.e., elevated BMI). Identifying such limitations can 


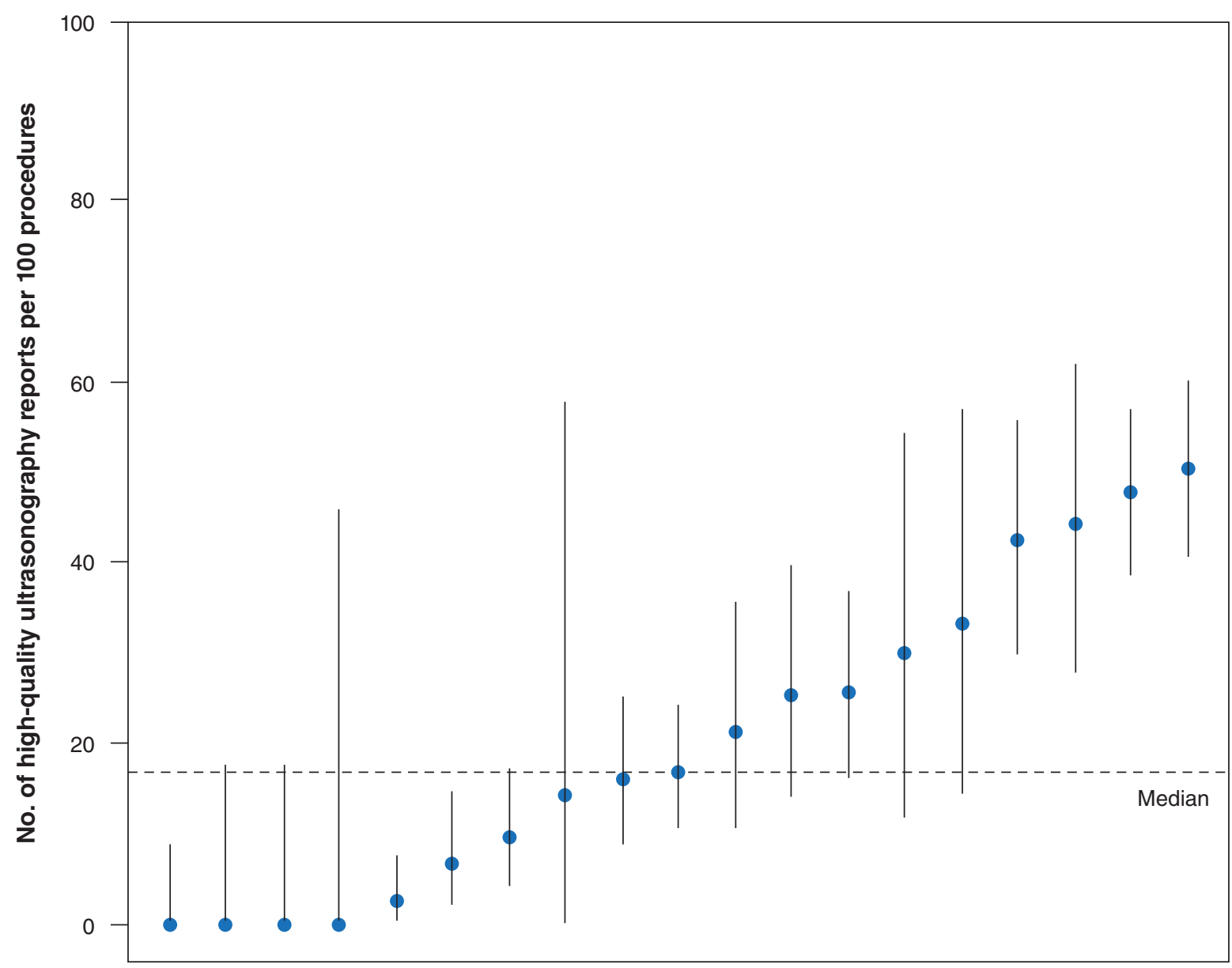

CAPTURE sites

Figure 2: Site-specific rates (in ascending order) of high-quality reporting of ultrasonography results (per 100 procedures). Note: Each bar represents an individual Canadian Women with Uterine Fibroids Registry (CAPTURE) site, the identity of which is kept anonymous. Each bullet point is the site estimate and the vertical line is a $95 \%$ confidence interval based on the exact binomial distribution. The horizontal dashed line is the overall (across all subjects) average.

help to identify focus areas for improvement. Furthermore, we suggest that national clinical practice guidelines for uterine fibroids should include guidance on choice of imaging modality and identify standards with respect to imaging quality for fibroid evaluation.

\section{Limitations}

The findings of our study must be interpreted within the context of study design. One of the challenges of using data from a noninterventional registry is that data may be available in a heterogeneous manner based on local practice patterns. Unfortunately, the registry did not collect data on imaging characteristics such as route of ultrasonography (transabdominal or transvaginal), the specialty of the reporting physician (radiologist or gynecologist) and whether a technologist was involved in obtaining the images. It would be important to evaluate these variables in detail in future research and before starting quality-improvement initiatives. The training received by gynecologists or radiologists who are performing sonographic imaging of uterine fibroids should also be evaluated and standardized in accordance with unified international guidelines. ${ }^{16,17}$

\section{Conclusion}

Our findings hold important implications for the evaluation and treatment of uterine fibroids. The results also shed light on optimizing resource allocation in the evaluation of this common gynecologic condition. Characteristics defined 
through high-quality imaging and standardized reporting may guide selection of medical versus surgical management of fibroids as well as guide surgical planning. Improper surgical planning may lead to suboptimal patient outcomes. This study shows that MUSA recommendations for evaluation using ultrasonography of fibroids are not being consistently followed in many sites across Canada. We suggest that there is a prompt need to evaluate and subsequently develop standardized guidelines for imaging of uterine fibroids in Canada.

\section{References}

1. Stewart EA, Cookson C, Gandolfo R, et al. Epidemiology of uterine fibroids: a systematic review. B7OG 2017;124:1501-12.

2. Baird DD, Dunson DB, Hill MC, et al. High cumulative incidence of uterine leiomyoma in black and white women: ultrasound evidence. Am 7 Obstet Gynecol 2003;188:100-7.

3. Vilos GA, Allaire C, Laberge PY, et al. The management of uterine leiomyomas. 7 Obstet Gynaecol Can 2015;37:157-78.

4. Dueholm M, Lundorf E, Hansen ES, et al. Accuracy of magnetic resonance imaging and transvaginal ultrasonography in the diagnosis, mapping, and measurement of uterine myomas. Am 7 Obstet Gynecol 2002;186:409-15.

5. Marret H, Fritel X, Ouldamer L, et al. Therapeutic management of uterine fibroid tumors: updated French guidelines. Eur 7 Obstet Gynecol Reprod Biol 2012;165:156-64.

6. Van den Bosch T, Dueholm M, Leone FP, et al. Terms, definitions and measurements to describe sonographic features of myometrium and uterine masses: a consensus opinion from the Morphological Uterus Sonographic Assessment (MUSA) group. Ultrasound Obstet Gynecol 2015;46:284-98.

7. Bedaiwy MA, Janiszewski P, Singh SS; CAPTURE Steering Committee. A patient registry for the management of uterine fibroids in Canada: protocol for a multicenter, prospective, noninterventional study. FMIR Res Protoc 2018;7:e10926.

8. Murji A, Bedaiwy M, Singh SS, et al. Influence of ethnicity on clinical presentation and quality of life in women with uterine fibroids: results from a prospective observational registry. 7 Obstet Gynaecol Can 2019 Dec. 24. [Epub ahead of print]. pii: S1701-2163(19)30960-0. doi: 10.1016/j.jogc.2019.10.031.

9. Coyne KS, Margolis MK, Bradley LD, et al. Further validation of the uterine fibroid symptom and quality-of-life questionnaire. Value Health 2012;15:135-42.

10. Spies JB, Coyne K, Guaou Guaou N, et al. The UFS-QOL, a new diseasespecific symptom and health-related quality of life questionnaire for leiomyomata. Obstet Gynecol 2002;99:290-300.

11. Ruta DA, Garratt AM, Chadha YC, et al. Assessment of patients with menorrhagia: How valid is a structured clinical history as a measure of health status? Qual Life Res 1995;4:33-40.

12. Larsen K, Merlo J. Appropriate assessment of neighborhood effects on individual health: integrating random and fixed effects in multilevel logistic regression. Am 7 Epidemiol 2005;161:81-8.

13. Kubik-Huch RA, Weston M, Nougaret S, et al. European Society of Urogenital Radiology (ESUR) guidelines: MR imaging of leiomyomas. Eur Radiol 2018;28:3125-37.
14. Pérez-Lopez FR, Ornat L, Ceausu I, et al. EMAS position statement: management of uterine fibroids. Maturitas 2014;79:106-16.

15. Hartmann KE, Fonnesbeck C, Surawicz C, et al. Management of uterine fibroids. Comparative Effectiveness Review No. 195. Rockville (MD): Agency for Healthcare Research and Quality; 2017.

16. Leonardi M, Murji A, D'Souza R. Ultrasound curricula in obstetrics and gynecology training programs. Ultrasound Obstet Gynecol 2018;52:147-50.

17. Abuhamad A, Minton KK, Benson CB, et al. Obstetric and gynecologic ultrasound curriculum and competency assessment in residency training programs: consensus report. Am $\mathcal{F}$ Obstet Gynecol 2018;218:29-67.

Affiliations: Department of Obstetrics and Gynaecology (Bougie), Queen's University, Kingston, Ont.; Department of Obstetrics and Gynecology (Bedaiwy), The University of British Columbia, Vancouver, BC; Department of Obstetrics and Gynecology (Laberge), CHU de Québec, Laval University, Quebec, Que.; Applied Health Research Centre (Lebovic), LKSKI, St. Michael's Hospital, Toronto, Ont.; Department of Obstetrics and Gynecology (Leyland), McMaster University, Hamilton, Ont.; Department of Medical Imaging (Atri), University of Toronto; Department of Obstetrics and Gynecology (Murji), Mount Sinai Hospital, University of Toronto, Toronto, Ont.

Contributors: All of the authors contributed to the conception or design of the work. Gerald Lebovic performed the data analysis for this study. Olga Bougie, Ally Murji and Mohamed Bedaiwy interpreted the data from the analysis. All authors were involved in drafting the manuscript and in the critical revisions for intellectual content, and gave final approval of the version to be published and agreed to be accountable for all aspects of the work.

Funding: The study was sponsored by Allergan, Markham, Ontario. Funding for editorial assistance was provided by Allergan plc, Dublin, Ireland and editorial assistance was provided to the authors by Natalie Prior (Complete HealthVizion). Neither honoraria nor payments were made for authorship. Allergan did not play a role beyond funding the study and editorial assistance.

Data sharing: Data reported in this manuscript is available within the article or its supplementary materials. Allergan will share de-identified patient-level data or study level data for studies registered at Clinical Trials.gov.

Acknowledgments: The authors thank the following members of the Canadian Women with Uterine Fibroids Registry (CAPTURE) Steering Committee: Drs. Sari Kives, George Vilos, Joshua Polsky and Liane Belland. This manuscript was reviewed by the CAPTURE Steering Committee, which also contributed to the development of the study goals.

Supplemental information: For reviewer comments and the original submission of this manuscript, please see www.cmajopen.ca/content/8/3/ E506/suppl/DC1. 